\title{
DESIGN OF CONTROL SYSTEM OF HUMAN-COMPUTER INTERACTIVE AGV ROBOT USING DOUBLE PHOTOELECTRIC ENCODER POSITIONING
}

\author{
Yang Shua ${ }^{1}$, Yi-qiao Shi ${ }^{1}$, Lan Xue ${ }^{1}$ \\ ${ }^{1}$ Huaian Vocational College of Information Technology, Huai'an 223003, China. \\ Email: shuaiyang22shy@126.com
}

\begin{abstract}
In order to study the design functional requirements of robot control system, in this study, an automated guided vehicle (AGV) robot is proposed, which can complete the autonomous navigation and handling of objects. Based on the design idea of "core board + bottom board", the power circuit, sensor signal acquisition circuit, drive control circuit, human-computer interaction module interface circuit and USB interface circuit are designed. The motion control algorithm based on double encoders is studied. Positioning is the premise of motion control, so the positioning algorithm based on double encoder is also derived. The results show that the motion control algorithm based on double encoders is verified by experiments, and the AGV robot control system designed meets the design functional requirements. In the experiment with the maximum speed of $1 \mathrm{~m} / \mathrm{s}$, the robot motion control effect is good, and the final positioning accuracy can reach within $2 \mathrm{~cm}$. It can be seen that the control system basically meets the design requirements, improves the dynamic response and greatly improves the positioning accuracy.
\end{abstract}

Keywords: AGV Robot; Positioning and Navigation; Control System; Path Planning.

\section{Introduction}

Robot technology has been widely used in aerospace, medical, education, service industry and other fields in the process of continuous development. In this process, robots become accessible to ordinary people [1].

The service robots in airports, restaurants and other places are getting closer and closer to people, so it is necessary for people to control the robots.

In this process, people are more concerned about the robot's understanding of human intention, so they put forward higher requirements for the robot's intelligence and security.

Human computer interaction has always been the focus of robotics research.

A successful interaction often depends on many factors, such as whether the interaction can be accepted by people, and whether the interaction can play a better role in a limited environment.

Therefore, in order to improve the social identity of human-computer interaction, the first problem to be solved is the robot's adaptation to human motion characteristics [2].

The action and posture of some humanoid robots are the important embodiment of the robot itself, which will also have a deep impact on the user.
Therefore, it is a challenging work to control the robot's body movement, and it is also an important topic in robot research [3].

The most intuitive way to achieve humancomputer interaction is to use imitation. In this process, the user plays the role of a teacher. By performing how to do a complete action, the robot repeats and learns the action through observation.

In this study, the basic hardware, software and algorithm in the control system of human-computer interactive automated guided vehicle (AGV) robot are studied.

Based on the control system, more functions can be realized. Because the control system can realize the functions of direct walking, turning and positioning, and meet the basic requirements of robot competition, it can be used in the competition.

At the same time, because it covers multidisciplinary knowledge, such as sensors, microcontrollers, control theory, electrical engineering, etc., it can also be used as a multidisciplinary and multi field teaching experiment platform.

In addition, the control system can be regarded as the bottom control platform of other robots (such as medical service robots, entertainment robots), based on which more complex functions can be developed. 


\section{Related Work \\ 2.1 Research progress abroad}

Hummel et al. (2019) proposed that UAVs are expected to operate autonomously, but they will also interact with humans to jointly solve tasks [4].

In order to support the civil UAV team, a distributed architecture is proposed.

The architecture can perform complex operations, such as image recognition, human coordination and flight control decision, not on UAV, but remotely.

The advantage of this architecture is that the computing power for image recognition is increased, and the human-computer interface can be integrated.

The downside is that it has to communicate, resulting in a delay in command reception. In the research, the design considerations of the distributed method, the example implementation on the smartphone and the application in the specific case of shelf inventory are discussed.

Khan et al. (2018) proposed to determine the opportunity to enhance user experience with semiautonomous social robots through user experience and human-computer interaction [5].

In this study, in order to use social robot partners in a specific public environment (such as a company's work area), it is prototype developed.

The challenge of this special environment has led to the need for more productive and comfortable offices for employees. At the same time, the availability, acceptability and feasibility of robot mate are evaluated.

The results emphasize that because HRI is highly interdisciplinary, the benefits of combining methods in other areas can benefit from meaningful social interaction with users.

\subsection{Domestic research progress}

Yang et al. (2019) proposed that gesture is one of the most important ways of human communication, which can effectively express the needs of users [6].

In the past few decades, gesture-based interaction has made great progress. Gesture interaction technology is introduced.

The definition and classification of gesture, input devices for gesture interaction and gesture interaction recognition technology are discussed. The application of gesture interaction technology in virtual reality has been studied, the existing problems of gesture interaction are summarized, and the future development is prospected.

Wong et al. (2019) proposed using a fuzzy system with appropriate rules to drive the server motor to achieve excellent performance [7].
The robot system is not only controlled by the interactive interface, but also realizes autonomous navigation by extracting appropriate map information.

The high sensory robot system obtains the interactive action through the combination of image recognition, appropriate path planning and obstacle detection technology to achieve the guiding goal.

In the implementation of hardware, the architecture of mobile robot and motor driver has been completely assembled to support home tasks.

The soft fuzzy system generates robust robot regulation to automatically achieve the perfect feasibility of obtaining and providing tasks.

\subsection{Summary of relevant research at home and abroad}

In conclusion, taking the positioning and navigation of dual photoelectric encoder as an example, the aim is to reduce the price of the humancomputer interactive AGV robot through certain hardware integration planning means, and improve the overall performance of the human-computer interactive autonomous robot handling and navigation subsystem through algorithms.

The two-dimensional code navigation method with high accuracy of single point positioning is used to realize the intelligent transportation function of the system.

In the running environment of the robot, a collision free path from the starting point to the target point is found, so that the cost of the robot running along the path to the end point is the least.

\section{Hardware Circuit Design of Control System for Human-Computer Interactive AGV Robot 3.1 Hardware structure of control system}

The hardware circuit is designed according to the functional requirements of human-computer interactive AGV robot. Based on the design idea of "core board + bottom board", the most basic hardware circuit has been realized on the core board, so only the hardware circuit on the bottom board needs to be designed.

The circuits on the bottom board mainly include: power circuit, reset circuit, sensor signal acquisition circuit, drive control circuit, human-computer interaction module interface circuit, and USB interface circuit.

The specific hardware structure is shown in figure 1. 


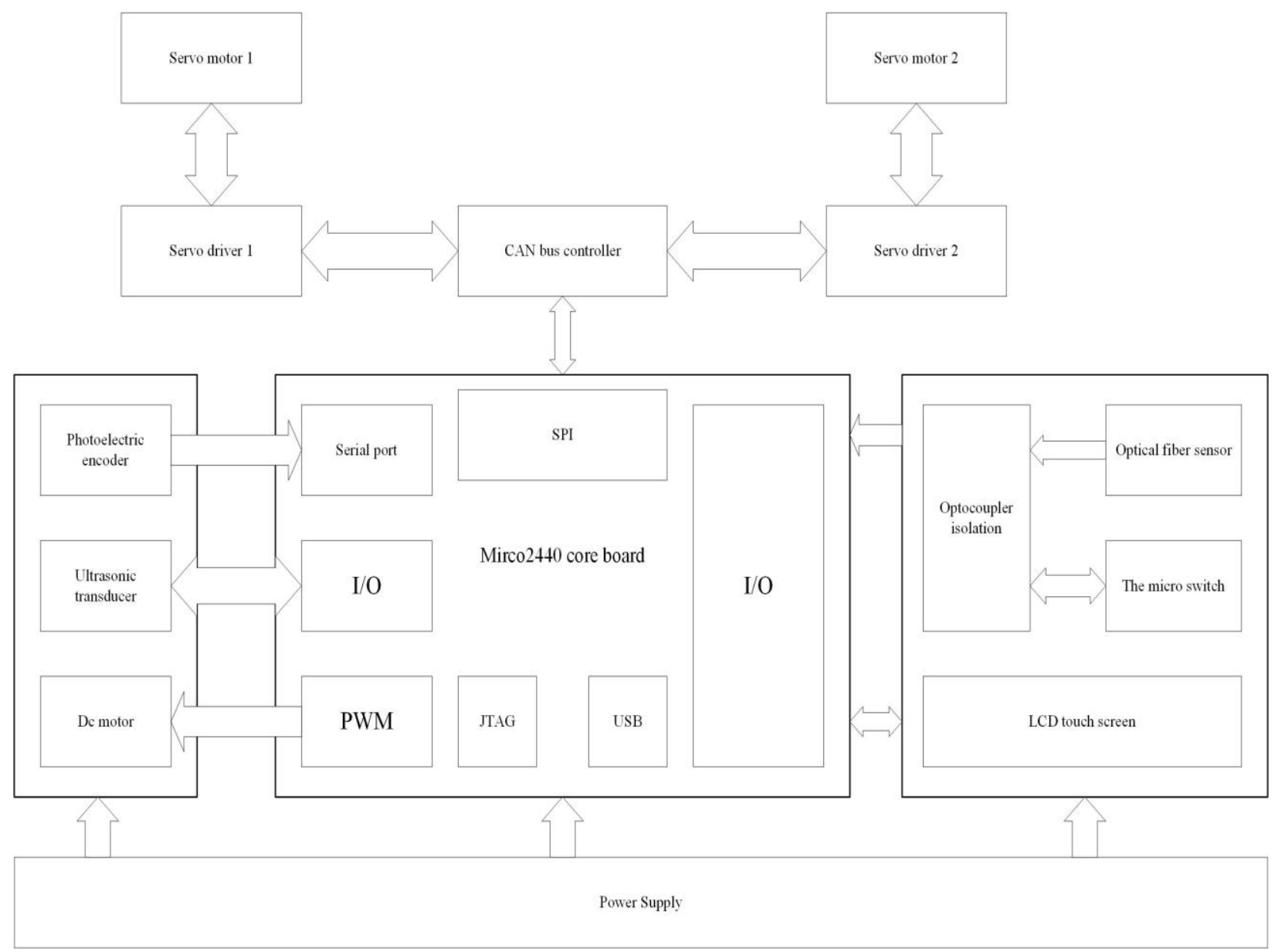

Figure 1: Hardware structure of control system

After the processor receives the corresponding signal and processes it, it drives the actuator to act, and the signal is detected by the sensor. According to the previous scheme, it is not difficult to find that the sensors required by the system include navigation related sensors, lift and grab positioning sensors.

According to different positioning and navigation methods, there are many commonly used sensors for indoor robots, including ultrasonic sensor, optical fibre sensor, electronic compass, gyroscope, photoelectric switch and photoelectric encoder [8]. In positioning, the most commonly used sensor is the photoelectric encoder, which is also used in this study. The detection of robot lifting and grasping needs to use lifting and grasping positioning sensors, usually a simple micro switch can meet the requirements.

The photoelectric encoder is mainly composed of photoelectric detector and grating disk. Its output signal is pulse or digital quantity, and the corresponding physical signal is mechanical geometric displacement. In the mechanical structure of the human-computer interactive autonomous navigation and handling robot designed by this research institute, the photoelectric encoder is installed coaxially with the left and right driving wheels and rotates at the same speed, and several pulse signals are detected and output by the photoelectric detection device.

As long as the number of pulses output by the photoelectric encoder in a unit time is known, the rotation distance of the left and right driving wheels of the robot can be calculated. Combined with the position and attitude of the robot, the function of dead reckoning can be realized [9]. In addition, the photoelectric encoder also provides two channels of optical code output for judging the direction of the motor. The phase difference between the two signals is just $90^{\circ}$.

The A-CHA solid axis photoelectric encoder selected in this system has a working voltage of 5$26 \mathrm{v}$, the number of pulses output in a rotation cycle, that is, the number of grating lines $\mathrm{Rn}$ is 3000 lines, and the maximum speed is $5000 \mathrm{r} / \mathrm{min}$.

Positioning accuracy is:

$$
\begin{aligned}
& A P=2 \times \pi \times r / R_{n} \\
& =2 \times 3.1415926 \times 0.05 / 3000 \\
& =0.105 \mathrm{~mm}
\end{aligned}
$$

In equation $1, \mathrm{r}$ represents the radius of the driving wheel of the robot.

The positioning accuracy of the system is less than $1 \mathrm{~mm}$, which can obviously be met. 
The maximum rotation speed that the photoelectric encoder can meet, as shown in equation 2:

$$
\begin{aligned}
& v_{\max }=2 \times \pi \times r \times 5000 / 60 \\
& =2 \times 3.1415926 \times 0.05 \times 5000 / 60 \\
& =26.18 \mathrm{~m} / \mathrm{s} \geq 1 \mathrm{~m} / \mathrm{s}
\end{aligned}
$$

It also meets the requirements. At the same time, when designing the system, the time Ts shall meet the following requirements:

$$
\begin{aligned}
& T_{s} \geq A P / v \\
& =0.105(\mathrm{~mm}) / 1(\mathrm{~m} / \mathrm{s}) \\
& =0.105 \mathrm{~ms}
\end{aligned}
$$

In order to realize the corresponding action of the control system, the actuator is essential.

Generally, the control signal is amplified by the driver and sent to the actuator. First of all, the premise of the system actuator with standard action is correct control signal. Secondly, the system must

\begin{tabular}{|c|c|}
\hline Parameters & Value \\
\hline The fastest walking speed of robot: $\mathrm{v}$ & $1 \mathrm{~m} / \mathrm{s}$ \\
\hline Total weight of robot and load: $\mathrm{M}$ & $25 \mathrm{Kg}$ \\
\hline Mechanical transmission efficiency: $\eta$ & 0.7 \\
\hline Friction coefficient between wheel and ground: $\mu$ & 0.3 \\
\hline Diameter of driving wheel: $\mathrm{D}$ & $0.1 \mathrm{~m}$ \\
\hline Gravitational constant: $\mathrm{G}$ & $9.8 \mathrm{~m} / \mathrm{s} 2$ \\
\hline
\end{tabular}
have reliable action and suitable actuator.

The selection of driving and executing mechanism needs to consider the relevant parameters of robot. The robot parameters are shown in table 1.

\subsection{Sensor signal acquisition circuit}

In the system, serial port is used to obtain the output signal of photoelectric encoder. Because S3C2440A contains a serial controller, only external level conversion chip and serial interface are needed.

The level conversion chip used in the design is MAX3232. RS232 is one of the main serial communication interfaces and belongs to the full duplex communication protocol.

In actual communication, only three external signal interfaces are needed: RXD, TXD and GND.
As the high- and low-level signals defined in TTL circuit of S3C2440A system are different from those defined in RS232 standard, the level comparison is shown in table 2 , so the two can communicate only after level conversion [10].

Table 2: Comparison of TTL level and RS232

\begin{tabular}{|l|l|l|}
\hline & TTL level & RS232 level \\
\hline 1 & $2 \sim 3.3 \mathrm{~V}$ & $-5 \sim-15 \mathrm{~V}$ \\
\hline 0 & $0 \sim 0.4 \mathrm{~V}$ & $+5 \sim+15 \mathrm{~V}$ \\
\hline
\end{tabular}

The circuit design is shown in figure 2.
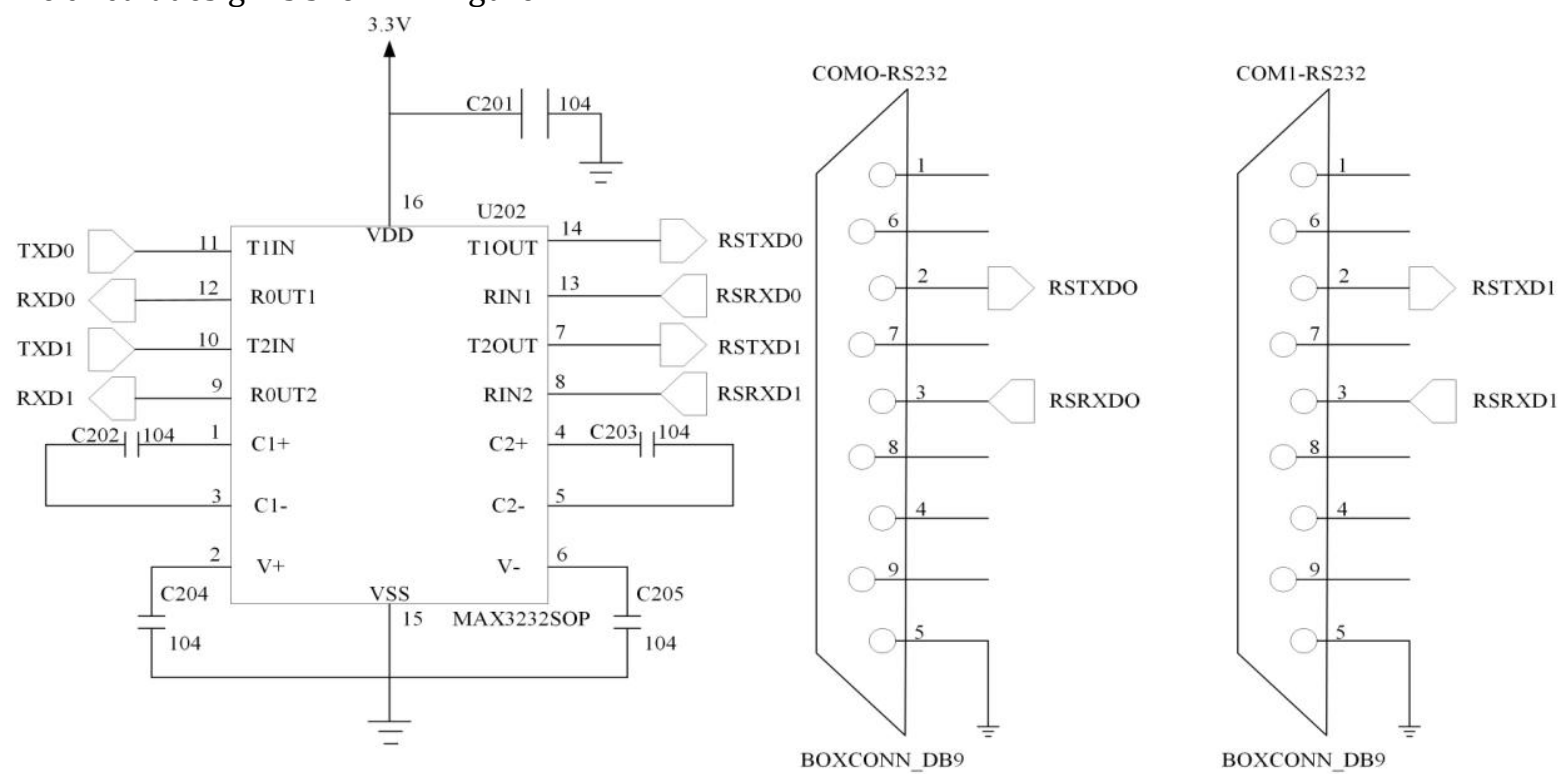

Figure 2: Photoelectric encoder interface circuit 


\subsection{Drive control system}

CAN bus is a communication protocol invented by BOSCH Company in Germany. The reference object is OSI model. It uses the physical layer, data link layer and application layer of OSI model, and is widely used in some industrial control fields such as automobile [11]. CAN bus has a long transmission distance, a large number of nodes, and multi master mode. All nodes in the same network have the chance to obtain the master node and send the command actively. CRC verification is provided. After the data transmission fails, it can be automatically retransmitted. When the network error is serious, the node exits automatically.

The message structure is short frame structure, which can reduce information transmission time and improve data correctness.

Because Elmo driver contains CAN bus interface, CAN bus and Elmo drive motor are used for data transmission in the design. However, S3C2440A is not integrated with CAN bus controller, so MCP2515, an independent bus controller with SPI interface, is used to expand CAN bus.

Among them, MCP2515 is connected with the processor through the SPI interface.

The chip selection signal of CAN controller uses the nSS0 pin of SPIO of S3C2440A, and the low level indicates effective. The GPG13 pin is connected to the / reset pin of MCP2515. The / INT pin of MCP2515 is connected to the EINT20 pin of the processor for interrupt generation. A $12 \mathrm{MHz}$ crystal oscillator is used to provide clock signal for MCP2515. PCA82C250 is used as the transceiver of CAN bus.

In the circuit design, CAN controller uses $3.3 \mathrm{~V}$ power supply, and PCA82C250 chip uses 5V power supply. Although the two chips have different working voltages, two data lines are used to communicate between them. For MCP2515 chip, one line is dedicated to output, the other line is dedicated to input, and the same is true for PCA82C250 chip [12].

Therefore, communication between them as long as one-way electrical characteristics can meet.

When $5 \mathrm{~V}$ power supply, the input high level range of PCA82C250 chip is $2 \mathrm{~V} \sim 5 \mathrm{~V}$, while the minimum high-level output of TXCAN pin of MCP2515 controller is $2.6 \mathrm{~V}$, so it can meet the system requirements and can be connected directly.

However, the output level of PCA82C250 pin cannot meet the requirements of MCP2515 for the high-level range of input pin, so the resistor R303 and R304 are used for voltage division to meet the requirements [13].
Based on the above analysis, the electrical standards of the two chip interfaces can be met.

S3C2440A contains USB controller inside, so it is led out in the design to realize USB communication.

The USB circuit in the system is mainly used to download image files.

The hardware circuit is shown in figure 3.

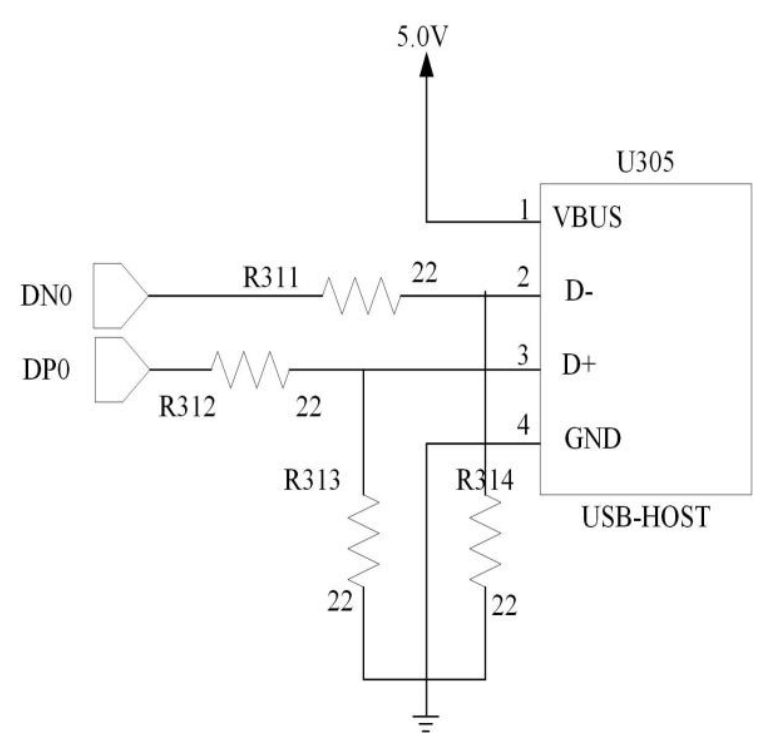

Figure 3: USB interface circuit

\subsection{Positioning algorithm based on double photoelectric encoder}

According to the mechanical structure of AGV robot, photoelectric encoder is installed on the coaxiality of the left and right driving wheels of the robot to realize the positioning function.

However, the output of the encoder represents the walking distance of the driving wheel, so it is necessary to combine the kinematic model of the robot to deduce the relationship between the position and the walking distance of the driving wheel.

$$
\begin{aligned}
& \bar{x}=\frac{1}{2}\left(v_{L}+v_{R}\right) \cos \theta \\
& =v \cos \theta \\
& \bar{y}=\frac{1}{2}\left(v_{L}+v_{R}\right) \sin \theta \\
& =v \sin \theta \\
& \bar{\theta}=\frac{1}{L}\left(v_{L}-v_{R}\right)
\end{aligned}
$$

By integrating the two sides of each equation in the above equation, it can be obtained that:

$$
\begin{aligned}
& x(t)=x(0)+\int_{0}^{t} v(t) \cos \theta(t) d t \\
& y(t)=y(0)+\int_{0}^{t} v(t) \sin \theta(t) d t
\end{aligned}
$$




$$
\theta(t)=\theta(0)+\int_{0}^{t} \frac{1}{L}\left(v_{L}(t)-v_{R}(t)\right) d t
$$

Where, $v_{L}(t)$ and $v_{R}(t)$ represent the speed of left and right driving wheels at time $t$ respectively. Because of the periodic collection of information, the periodic discretization equation is as follows:

$$
\begin{aligned}
& x_{n+1}=x_{n}+\int_{t_{n}}^{t_{n+1}} v(t) \cos \theta(t) d t \\
& y_{n+1}=y_{n}+\int_{t_{n}}^{t_{n+1}} v(t) \sin \theta(t) d t \\
& \theta_{n+1}=\theta_{n}+\int_{t_{n}}^{t_{n+1}} \frac{1}{L}\left(v_{L}(t)-v_{R}(t)\right) d t \\
& =\theta_{n}+\frac{1}{L}\left(\Delta S_{L, n}-\Delta S_{R, n}\right)
\end{aligned}
$$

In the above equation, the pose information of the robot at tn time is $(x n, y n, \theta n) T$, and that at $t n+1$ time is $(\mathrm{xn}+1, \mathrm{yn}+1, \theta \mathrm{n}+1) \mathrm{t} . \Delta S_{L, n}$ and $\Delta S_{R, n}$ represent the walking distance from tn time to $t n+1$ time.

\section{Design and Research of AGV Robot Control System 4.1 Design of human-computer interaction module}

In the design process of large-scale control system, it has a perfect human-computer interface, which is convenient for workers to implement better debugging and control of the control system.

Therefore, this module is also introduced in this study. In the design and implementation of humancomputer interaction, the choice of graphic library is very important [14].

In the application process of wheeled robot, due to the different environments and tasks of the robot, the selection of graphics library must also meet these changes. For example, GUI needs to save system resources, have good enough performance and stability.
MiniGUI is a kind of software with superior performance and strong applicability developed by Chinese people independently. Its main application scope is the multi window graphics operating system of embedded Linux system. However, any operating system that supports POSIX threads can run.

Microwindows, like Linux, is a GUI image library software that publishes source code. Its graphic interface is not only practical and beautiful, but also has some advantages of modern GUI system. It is also very compatible with most of the control chips in the market. But unfortunately, for various reasons, its driver module is not perfect.

OpenGUI has excellent built-in function modules and can be programmed with $\mathrm{C}++$. In addition, it is also compatible with many peripheral event modules, and its application is simple and fast [15].

However, it can only be applied to x86 architecture, which doomed its limitations in the field of robotics, because most of the time, the main controller is only ARM or other microprocessors, rather than $\mathrm{x} 86$ architecture processors.

\subsection{Motion subprogram design based on double encoder}

First, according to the task requirements of the system, a planned path from the starting point to the final target point is divided into a small path.

Then, three arrays are generated to save the parameters of the start point and end point of each path, i.e. abscissa, ordinate and attitude angle.

The design flow of motion subprogram is shown in figure 4.

Among them, flag indicates the flag bit of completing the task. When the system enters the motion subroutine, it first determines whether it has reached the final target point (that is, whether the flag is equal to 1 ).

If the flag is equal to 1 , it means that the current task has been completed, and it just returns. If the flag is not equal to 1 , first, it is necessary to update the current target point $\mathrm{B}(\mathrm{x} 2, \mathrm{y} 2, \theta 2)$ and calculate the displacement increment of the left and right encoders according to the output pulse number of the encoders. 


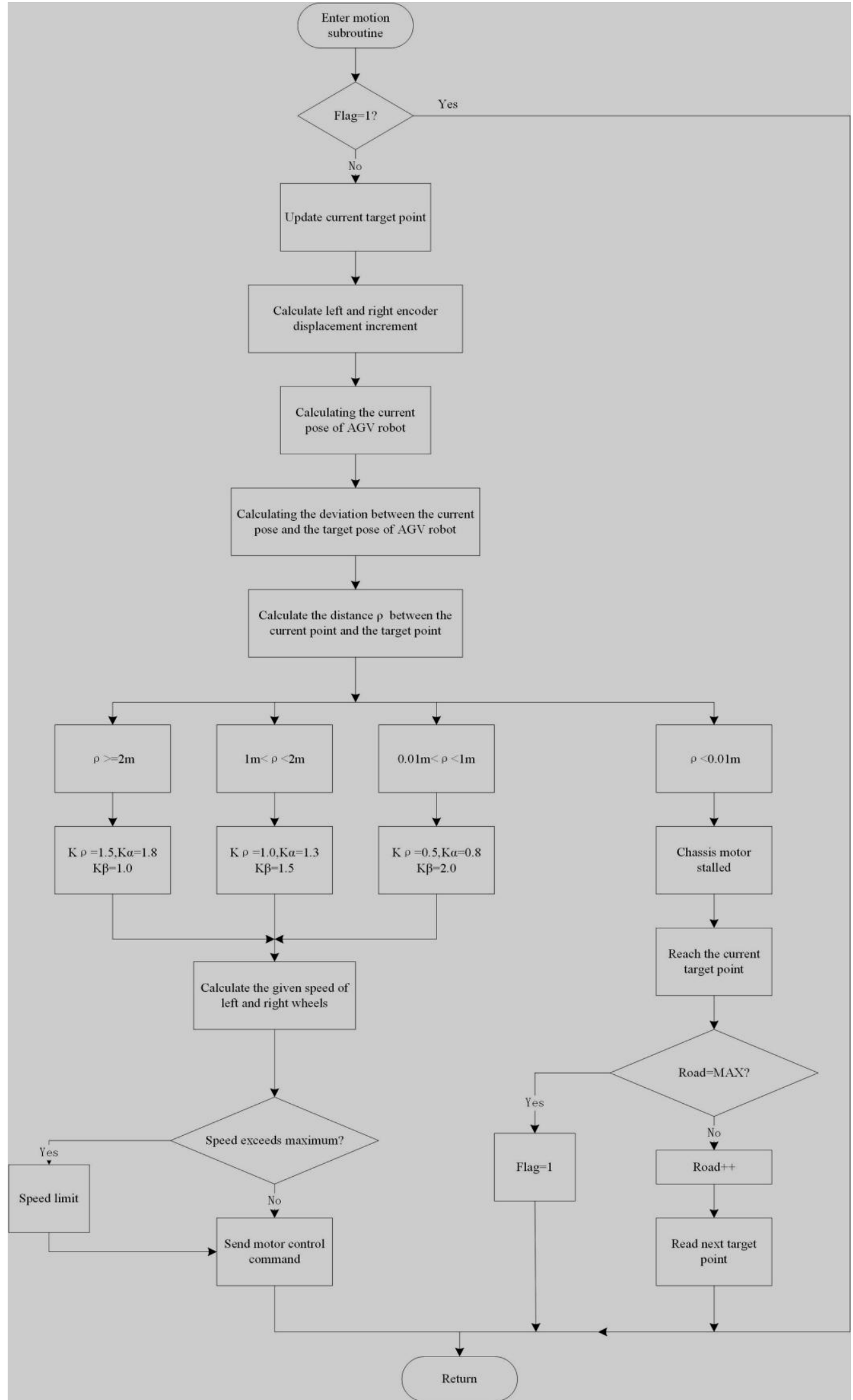

Figure 4: Flow chart of motion subprogram design

According to the track estimation, the current pose a $(x 1, y 1, \theta 1)$ of AGV robot is updated. Then, the deviation between the current pose and the target pose of AGV robot is calculated. Different $K \rho, K \alpha, K \beta$ can be set according to the value of $\rho$ to get the given speed of the left and right wheels, and check whether 
the calculated speed is less than the maximum speed allowed by the motor. Then, the speed command is sent to the two motors of the chassis through CANopen mode. Then, after $10 \mathrm{~ms}$ of the program, the next cycle is started. If the robot reaches the target point of the small path, but does not reach the target point of the whole path, the robot enters the next cycle after $10 \mathrm{~ms}$, and approaches the next target point.

Until the current target point reached by the robot coincides with the final target point, the flag is set to 1 . In the next program cycle, it is necessary to just go back directly.

Table 3. Track error record ( $\left.\mathrm{mm}, \mathrm{mm},{ }^{\circ}\right)$

\begin{tabular}{|c|c|c|c|}
\hline Coordinate point No. & Experiment 1 & Experiment 2 & Experiment 3 \\
\hline 1 & $0,0,0$ & $0,0,0$ & $0,0,0$ \\
\hline 2 & $20,-30,-1$ & $-20,-50,3$ & $-50,40,4$ \\
\hline 3 & $30,0,1.5$ & $60,15,6$ & $30,50,-2$ \\
\hline 4 & $15,20,2.2$ & $-30,50,0$ & $30,20,3.2$ \\
\hline 5 & $0,30,-1.3$ & $-20,40,4$ & $30,40,-5.5$ \\
\hline 6 & $30,30,-0.1$ & $50,40,-0.4$ & $25,30,-2$ \\
\hline 7 & $25,30,0.2$ & $15,20,-0.6$ & $20,18,0.4$ \\
\hline 8 & $10,20,4.1$ & $0,15,5.1$ & $16,10,5$ \\
\hline
\end{tabular}

It can be seen from table 3 that in the experiment with the maximum speed of $1 \mathrm{~m} / \mathrm{s}$, the maximum deviation of the control system is only $6 \mathrm{~cm}$, the maximum attitude error at the turning is within $6^{\circ}$, and the final positioning accuracy is within $2 \mathrm{~cm}$.

\subsection{Motion subprogram design based on tracing}

AGV robot collects real-time data through optical fibre sensor when it is tracking and walking. After the data is collected, it is processed accordingly. The time interval of collecting data is $0.5 \mathrm{~ms}$. Once five data are collected, they are filtered. The idea of filtering is: traverse 5 collected data, judge the occurrence times of 0 and 1 in these 5 data, and take the one with more occurrence times as the sampling value of this time.

Figure 5 shows the procedure flow.

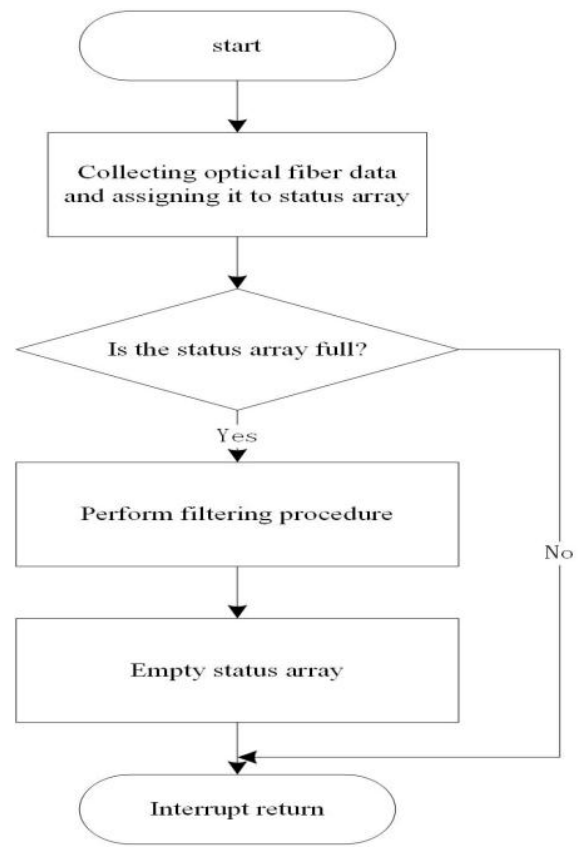

Figure 5: Optical fibre acquisition program flow
When the AGV robot is tracking and walking, the signal is collected by the optical fibre sensor, and then the collected signal is sent to the processor.

After receiving the signal, the processor processes it and generates the corresponding control signal, which is used to ensure the accuracy of AGV robot tracking and walking.

The procedure flow is shown in figure 6.

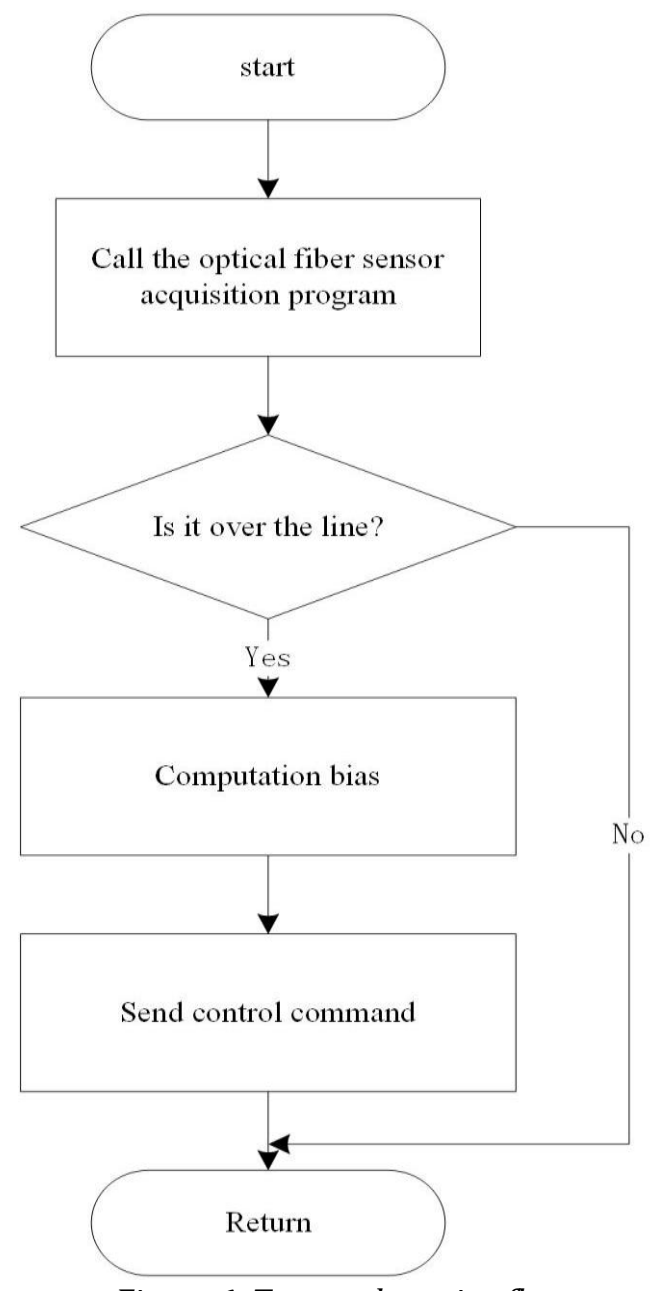

Figure 6: Trace subroutine flow 


\subsection{Subprogram design of grabbing objects}

The machine hand (gripper) and the machine arm (guide rail) have one motor to control respectively. The tasks of machine hand clamping and placing depend on the machine hand motor, which needs to control the forward and reverse rotation of the motor. The power source of machine hand lifting is the machine arm, which also needs to control its forward and reverse rotation.
Therefore, two PWM ports send signals to control the rotation speed of two motors respectively, and two GPIO ports control the rotation direction of two motors respectively. By detecting the signal of the micro switch of the machine arm, the machine hand can be controlled to a certain position. The PWM port can drive the motor to control the grasping action of the machine hand.

The specific process of grabbing objects is shown in figure 7.

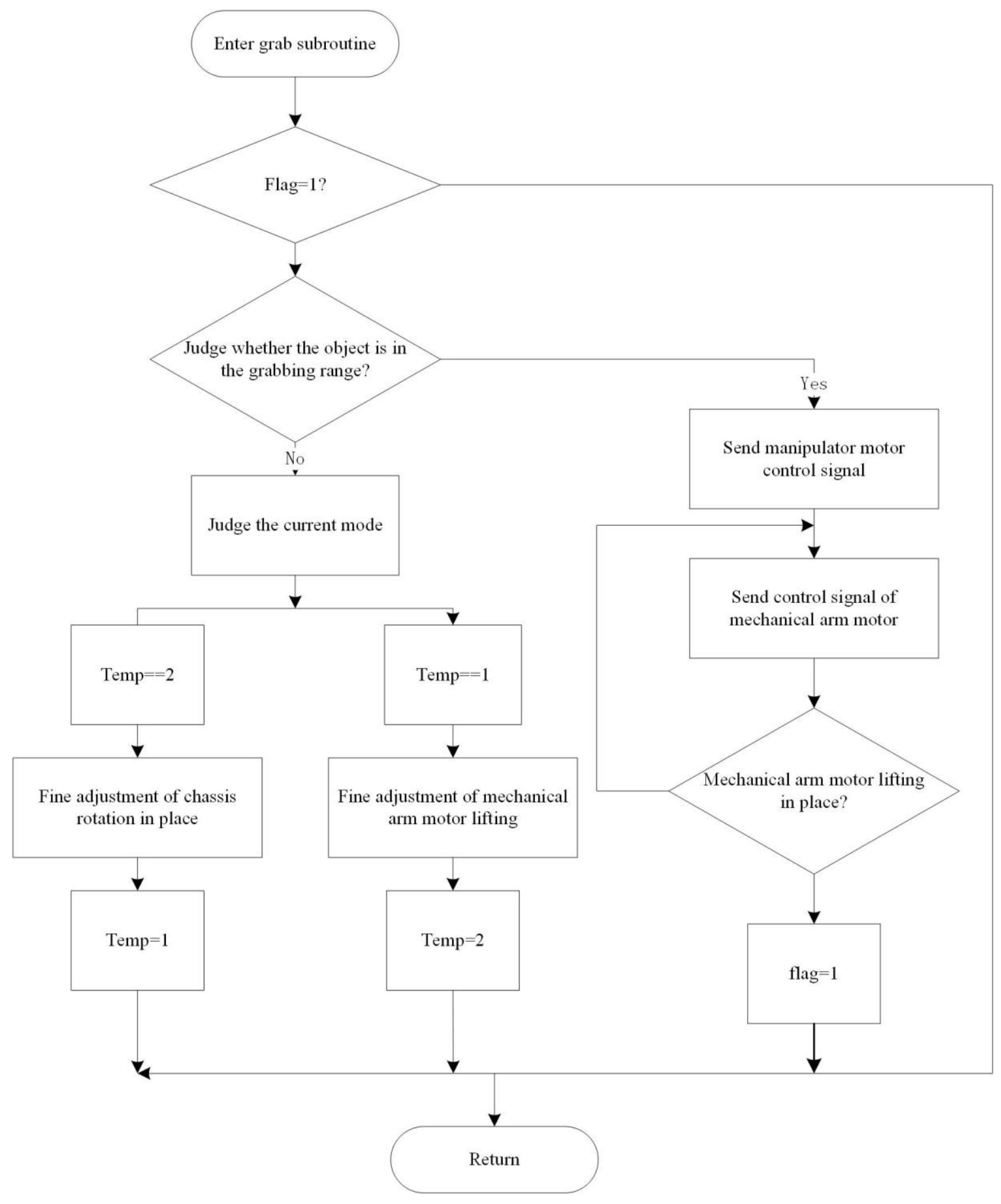

Figure 7: Object grabbing subroutine flow 


\section{Conclusions}

In this study, the control system of AGV robot is designed based on the function requirements of AGV robot. The control system takes ARM9 as the hardware platform, which has certain portability. By analysing the function requirements of AGV robot control system, the mechanical structure and overall scheme of the control system are determined.

The control system is divided into five modules: human-computer interaction module, drive control and actuator module, processor module, sensor module, power module, and then completed the selection of each module device. A motion control algorithm based on double encoders is proposed. In the motion control algorithm, a subsection control strategy is introduced to control the speed of the left and right wheels of the robot. In the experiment with a maximum speed of $1 \mathrm{~m} / \mathrm{s}$, the robot has a good tracking effect.

In all experiments, the maximum offset is only $6 \mathrm{~cm}$, and the final positioning accuracy is within $2 \mathrm{~cm}$. There is no doubt that robots will become more and more common in the future social life.

At the same time, the function of robot will be more and more perfect. Intellectualization is the development trend of wheeled robots. In this study, although the control system of the wheeled robot is designed, the design requirements are basically realized.

However, in order to develop a more intelligent robot with higher technology content, it is necessary to improve the motion control algorithm of AGV robot, so that the robot can complete tasks more quickly and accurately, such as introducing intelligent algorithms like fuzzy algorithm and genetic algorithm, so as to enhance the learning ability of the robot.

The path planning algorithm in this study is based on the known invariant environment information. In most cases, the environment is unknown or even changing, so the path planning algorithm in the dynamic unknown environment can be further studied.

\section{References}

[1] L. Wang, X. Fang, Q. Jia, "Radiation source intelligent handling system development," IOP Conference Series: Materials Science and Engineering, IOP Publishing, vol. 490, no. 7, pp. 072038, 2019.

[2] C. H. Chen, M. C. Wu, C. C. Wang, "Cloud-based Dialog Navigation Agent System for Service Robots," Sensors and Materials, vol. 31, no. 6, pp. 1871-1891, 2019.
[3] M. Fun, "Human-drone interaction: let's get ready for flying user interfaces," Interactions, vol. 25, no. 3, pp. 78-81, 2018.

[4] K. A. Hummel, M. Pollak, J. Krahofer, "A Distributed Architecture for Human-Drone Teaming: Timing Challenges and Interaction Opportunities," Sensors, vol. 19, no. 6, pp. 1379, 2019.

[5] S. Khan, C. Germak, "Reframing HRI Design Opportunities for Social Robots: Lessons Learnt from a Service Robotics Case Study Approach Using UX for HRI," Future Internet, vol. 10, no. 10 , pp. 101, 2018.

[6] L. I. Yang, J. HUANG, T. Feng, et al., "Gesture interaction in virtual reality," Virtual Reality \& Intelligent Hardware, vol. 1, no. 1, pp. 84-112, 2019.

[7] C. C. Wong, H. C. Chen, C. T. Lee, et al., "High interactive sensory robot system design in the indoor autonomous services applications," Journal of Intelligent \& Fuzzy Systems, vol. 36, no. 2, pp. 1259-1271, 2019.

[8] S. Muhammad, N. Mohammad, A. Bashar, et al., "Designing human assisted wireless sensor and robot networks using probabilistic model checking," Journal of Intelligent \& Robotic Systems, vol. 94, no. 3-4, pp. 687-709, 2019.

[9] S. K. Pathi, A. Kristoffersson, A. Kiselev, et al., "FFormations for Social Interaction in Simulation Using Virtual Agents and Mobile Robotic Telepresence Systems," Multimodal Technologies and Interaction, vol. 3, no. 4, pp. 69, 2019.

[10] E. González, F. A. A. Cheein, "Preliminary results on reducing the workload of assistive vehicle users: A collaborative driving approach," International Journal of Social Robotics, vol. 10, no. 5, pp. 555-568, 2018.

[11] C. Werner, G. P. Moustris, C. S. Tzafestas, et al., "User-oriented evaluation of a robotic rollator that provides navigation assistance in frail older adults with and without cognitive impairment," Gerontology, vol. 64, no. 3, pp. 278-290, 2018.

[12] A. Nourmohammadi, M. Jafari, T. O. Zander, "A survey on unmanned aerial vehicle remote control using brain-computer interface," IEEE Transactions on Human-Machine Systems, vol. 48, no. 4, pp. 337-348, 2018.

[13] K. Liu, R. Mulky, "Enabling autonomous navigation for affordable scooters," Sensors, vol. 18, no. 6, pp. 1829, 2018.

[14] E. Deng, B. Mutlu, M. J. Mataric, "Embodiment in socially interactive robots," Foundations and Trends $₫$ in Robotics, vol. 7, no. 4, pp. 251-356, 2019.

[15] M. K. Shahin, A. Tharwat, T. Gaber, et al., "A wheelchair control system using humanmachine interaction: Single-modal and multimodal approaches," Journal of Intelligent Systems, vol. 28, no. 1, pp. 115-132, 2019. 